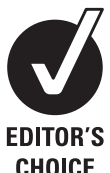

CHOICE

\title{
Imaging in acute ischaemic stroke: essential for modern stroke care
}

\author{
Daniel J Warren, ${ }^{1}$ Rachel Musson, ${ }^{1}$ Daniel J A Connolly, ${ }^{1}$ Paul D Griffiths, ${ }^{2}$ \\ Nigel Hoggard ${ }^{2}$
}

${ }^{1}$ Radiology Department, Royal Hallamshire Hospital, Sheffield NHS Teaching Hospitals Trust, Sheffield, UK

${ }^{2}$ Academic Unit of Radiology, University of Sheffield, Sheffield, UK

\section{Correspondence to}

Dr Daniel J Warren, C Floor, Department of Radiology, Royal Hallamshire Hospital, Glossop Road, Sheffield S10 2JF, UK; daniel.warren@sth.nhs.uk

Received 26 January 2010 Accepted 25 April 2010

\section{ABSTRACT}

Stroke is the second most common cause of death worldwide and the third most common in the UK. 'Time is brain' in ischaemic stroke; early reperfusion has been shown to lead to improved clinical outcomes, yet the majority of patients with acute stroke do not attend in time for thrombolysis as it is currently licensed, hence the interest in trials extending the therapeutic window. Defining the ischaemic penumbra is of crucial importance in choosing the appropriate patients for thrombolytic therapy who attend outside the optimal therapeutic window. Integrated stroke imaging, including demonstration of potentially salvageable tissue with either MR perfusion/diffusion studies or CT perfusion, is increasingly likely to play a central role in future management strategies and widening of the potential therapeutic window. This review highlights the basic imaging findings of acute stroke and discusses the role of advanced CT and MR techniques as well as options for vascular imaging.

\section{INTRODUCTION}

Stroke is defined as 'a clinical syndrome consisting of rapidly developing clinical signs of focal (or global in the case of coma) disturbance of cerebral function lasting more than $24 \mathrm{~h}$ or leading to death with no apparent cause other than a vascular origin'. ${ }^{2}$ Stroke is the second most common cause of death worldwide and the third most common in the UK after heart disease and cancer. It is the leading cause of severe adult disability in the UK, affecting over 100000 people in England each year, with significant emotional, physical and mental sequelae. ${ }^{3}$ The financial burden on the health system is immense, costing the NHS alone $£ 2.8$ billion per annum. ${ }^{1}$

The goal of early brain imaging is to exclude intracranial haemorrhage, identify ischaemic change, and exclude stroke mimics. Imaging also allows assessment of the intracranial and extracranial vasculature and facilitates delineation of the status of cerebral perfusion, demonstrating the infarct core and also the penumbra (potentially salvageable parenchyma), the identification of which may aid future management strategies.

Stroke can be subclassified into two major categories: ischaemic (accounting for $\sim 80 \%$ of all acute stroke events) and haemorrhagic (20\%). This review will focus on imaging of ischaemic stroke; haemorrhagic stroke (such as secondary to subarachnoid haemorrhage, subdural or extradural haemorrhage) is not further addressed in this review.
Ischaemic stroke encompasses multiple aetiologies including thrombosis, embolism, venous thrombosis and systemic hypoperfusion. Thrombolytic therapy with recombinant tissue plasminogen activator (rt-PA) is the treatment of choice for ischaemic stroke presenting within $3 \mathrm{~h}$ of clinical onset, provided that there is no contraindication to this treatment, including exclusion of intracranial haemorrhage by CT. Using a $3 \mathrm{~h}$ time period cut-off for thrombolysis, the NINDS trial ${ }^{4}$ showed efficacy, with the number needed to treat to benefit one patient being eight; however, there was a $6.4 \%$ treatment-related incidence of intracranial haemorrhage. A narrow time window for patient presentation, assessment and start of therapy results in only a small proportion of eligible patients receiving thrombolysis. Consequentially, fewer than $10 \%$ of patients with acute stroke actually receive rt-PA; in the NINDS trial, only $4 \%$ of patients met criteria for alteplase rt-PA. The European Medicines Agency granted approval of alteplase in $2002 .^{5}$

'Time is brain' is a frequently used idiom; the sooner a diagnosis of acute ischaemic stroke is made and thrombolysis treatment instigated (where indicated), the better is the patient outcome. ${ }^{1}$ Much of the literature addresses licensed use of alteplase rt-PA within $0-3 \mathrm{~h}$ of symptom onset; recently published data from the third European Cooperative Acute Stroke Study (ECASS III) lends support to the efficacy of rt-PA alteplase, and shows that extension of the treatment window to $4.5 \mathrm{~h}$ is efficacious in a large subgroup of patients. ${ }^{5}$ There is an argument that the therapeutic window should be widened, with decisions to treat being guided by improved and integrated imaging strategies. ${ }^{7}$

Optimisation of management of patients with acute stroke relies on prompt clinical assessment and stroke diagnosis followed by urgent brain imaging. In the UK in 2008, the National Institute for Health and Clinical Excellence (NICE) produced guidelines for diagnosis and initial management of acute stroke and transient ischaemic attack. ${ }^{1}$ These guidelines recommend that patients with acute stroke with indications for thrombolysis or early anticoagulation treatment, receiving anticoagulant treatment, who have a known bleeding diathesis, depressed level of consciousness, unexplained progressive or fluctuating symptoms, neck stiffness, fever or papilloedema, or severe headache at onset of stroke symptoms should undergo immediate (next scan slot and definitely within $1 \mathrm{~h}$ ) brain imaging. ${ }^{1}$ All other patients with stroke should receive brain imaging within $24 \mathrm{~h}$ of symptom onset. 
In this review, we will outline the characteristic imaging features of acute ischaemic stroke on both non-contrast CT and MRI. The role of advanced CT/MRI techniques including angiography and perfusion will be discussed, as well as options for vascular assessment, with consideration of both the intracranial and extracranial vasculature.

\section{NON-CONTRAST CT IMAGING}

CT is the imaging technique of choice in many institutions for the initial assessment of suspected stroke. It is readily available and permits rapid assessment of patients with acute stroke. Multidetector technology permits image acquisition within seconds and at sub-millimetre image resolution. ${ }^{7}$ Non-contrast CT allows detection of intracranial haemorrhage with a high degree of sensitivity, identification of stroke mimics, and also identification of early parenchymal ischaemic change.

Major current guidelines on the use of thrombolysis include non-contrast CT as an acceptable investigation from which to make a decision about the benefits of thrombolysis. The ECASS III major inclusion criteria were acute ischaemic stroke, age 18-80 years, stroke symptoms present for at least 30 min with no significant improvement before the start of lysis. The major exclusion criteria included intracranial haemorrhage and severe stroke as assessed clinically (a National Institutes of Health Stroke Scale score $>25$ (range $0-42$, with the higher score indicating more severe cerebral infarct and neurological impairment)) and severe stroke as assessed by imaging (defined as a stroke involving more than one-third of the middle cerebral artery (MCA) territory). ${ }^{5}$

Approximately three-quarters of all ischaemic cerebral infarcts occur within the territory supplied by the MCA. Detection of early ischaemic change on non-contrast CT can be difficult, with many of the early radiological features being subtle. A systematic review by Wardlaw and Mielke, ${ }^{8}$ which included assessment of observer reliability in detecting these early radiological signs, identified a mean sensitivity of $66 \%$ (range $20-87 \%$ ) and specificity of $87 \%$ (range $56-100 \%$ ) for detection of early infarction signs with CT; observer experience improved detection. Early radiological signs relate to parenchymal infarction and the cellular sequelae and also pathological vessel occlusion. ${ }^{8}$ Acute cerebral infarction results in cerebral hypoperfusion and cytotoxic oedema; an abrupt alteration and reduction in cellular

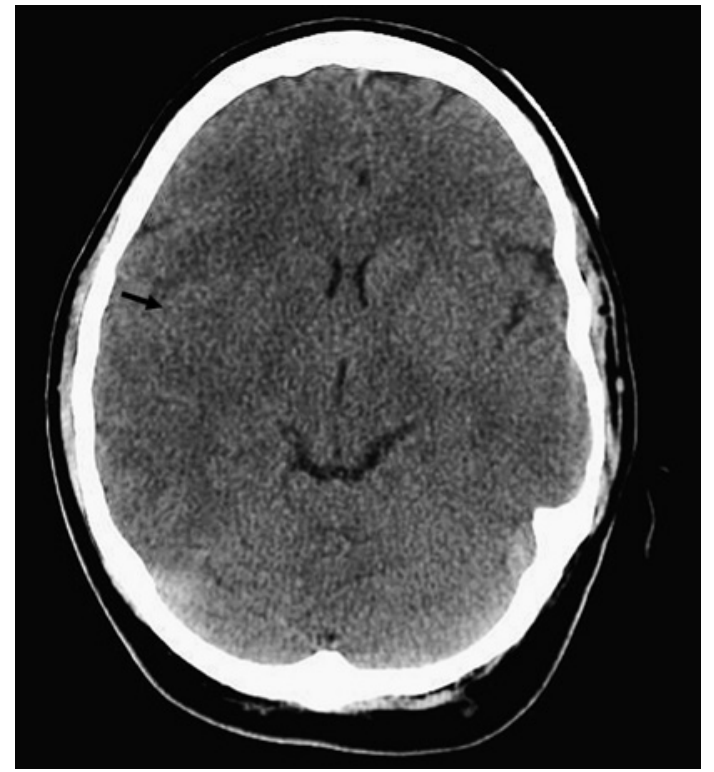

Figure 1 Non-contrast CT showing a large acute right middle cerebral artery infarct with diffuse right hemispheric swelling manifest by sulcal effacement and obliteration of the right sylvian fissure (black arrow).

oxygen and glucose supply leads to rapid failure of the sodium/ potassium pump, with a resultant shift of water from the extracellular to intracellular space and subsequent cytotoxic oedema.

The CT manifestation of this compartmental water shift is a focal mass effect with local cortical/gyral swelling and sulcal effacement (figure 1); furthermore, loss of the grey/white interface evolves. The most common areas of cerebral hypoattenuation identified are in the MCA territory, within the lentiform nucleus (figure 2), ${ }^{9}$ insular ribbon (figure 3), ${ }^{10}$ cerebral cortex and basal ganglia (figure 4). Delineation of early ischaemic change can be accentuated by use of variable window width and centre level settings to maximise the parenchymal contrast (figure 4A,B). ${ }^{11}$ A common finding on the non-contrast scan in the hyperacute phase is a normal study, and, as such, the earlier these ischaemic parenchymal changes are evident, the more severe is the degree of ischaemia. ${ }^{7}$
Figure 2 Subtle low attenuation within the right lentiform nucleus on non-contrast CT performed $3 \mathrm{~h}$ after sudden-onset left-sided weakness consistent with an acute right middle cerebral artery territory infarct $(A)$; follow-up scan at $24 \mathrm{~h}(\mathrm{~B})$ shows clear hypoattenuation within the right lentiform nucleus and within the posterior right frontal lobe.
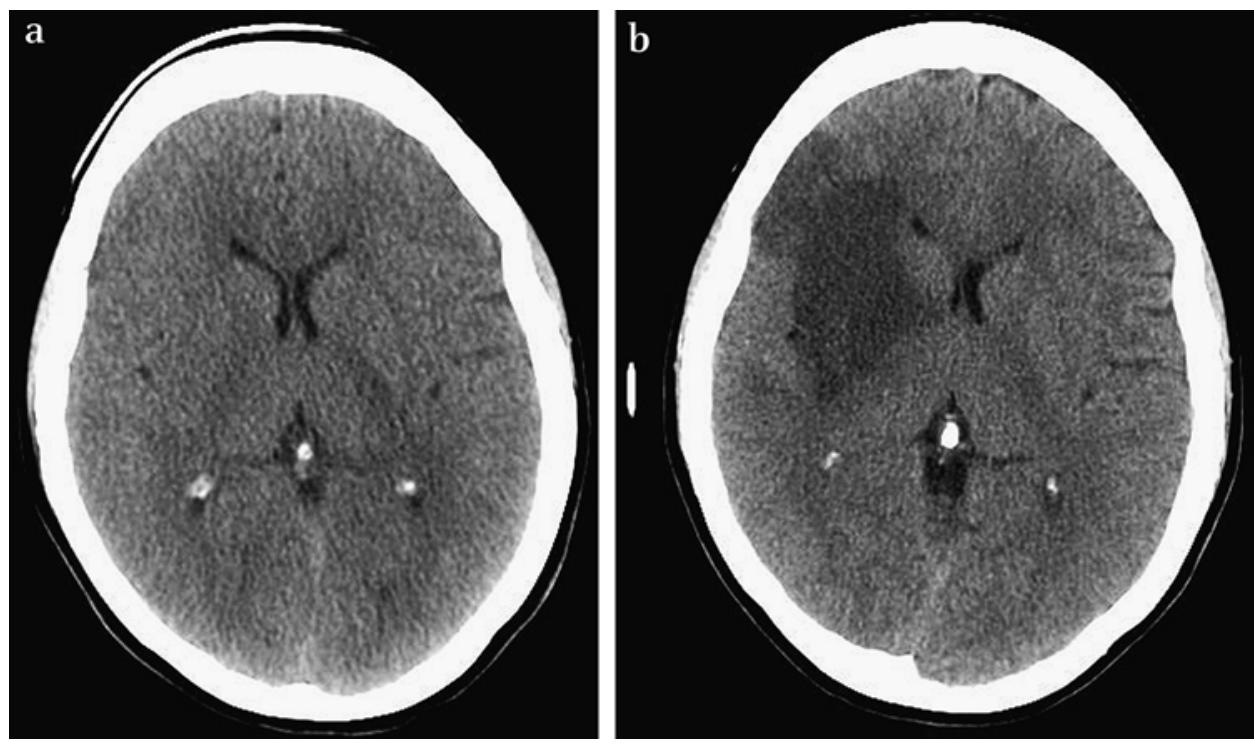


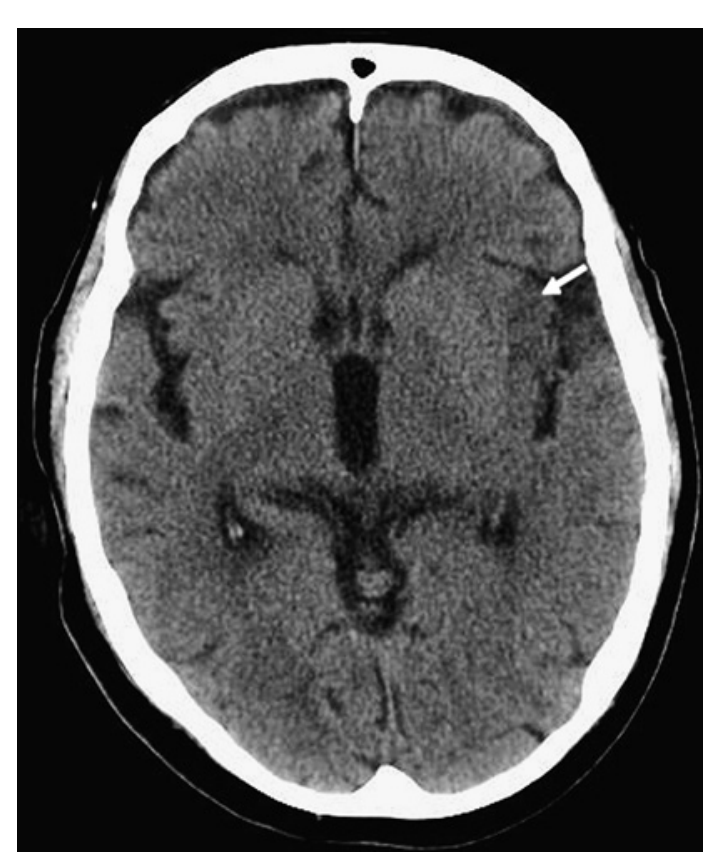

Figure 3 Hypoattenuation with the left insular ribbon in an acute left middle cerebral artery infarct (white arrow).

ECASS I highlighted that early evidence of cortical hypoattenuation affecting over one-third of the MCA territory was a predictor of poor outcome after intravenous thrombolysis within $6 \mathrm{~h}$ of stroke onset, with increased risk of symptomatic intracranial haemorrhage. ${ }^{12}$ The Alberta Stroke Program Early CT (ASPECT) score allows a more reliable assessment tool for estimation of infarct extent within the MCA territory, within $3 \mathrm{~h}$ of symptom onset, and also serves as a prognostic indicator. ${ }^{13-17}$ The ASPECT score is a validated semiquantitative scoring system whereby the MCA territory is divided into 10 regions of interest. The overall score is formulated by point deduction depending on the number of areas displaying early ischaemic change; thus a normal brain scan would score 10 points, and a score of zero indicates evidence of extensive ischaemic change throughout the MCA territory. ${ }^{14}{ }^{17}$ An ASPECT score of $>7$ is associated with improved post-thrombolysis outcome. $^{15}$

A further important sign that can be identified on the noncontrast CT study is vessel occlusion, seen as hyperdensity within the lumen of the vessel-'hyperdense MCA sign'. This is most often identified as linear hyperdensity within the proximal M1 segment of the MCA (figure 5A) or as a hyperdense 'dot' within the sylvian fissure (figure $5 \mathrm{~B}$ ). ${ }^{7}{ }^{18}$ Hyperdensity can be detected within any occluded vessel, and another example encountered quite commonly is thrombosis of the basilar artery (figure 6), although identification of this sign can be difficult secondary to vessel wall calcification and lack of a paired vessel for comparison. Hyperdensity may reflect flow stasis distal to a thrombus or the thrombus per se.

\section{MRI}

MRI can be used as an alternative or an adjunct to non-contrast CT, as much of the information is complementary to the noncontrast CT study. CT has the benefit of being readily available, inexpensive and fast; however, increasingly there is provision of out-of-hours MRI, continued sequence refinement leading to reduced MRI acquisition time, and improved sensitivity in detection of acute ischaemic change over non-contrast CT, especially in detection of early ischaemic change and identification of small infarcts and those within the posterior fossa. MRI provides improved anatomical detail and does not use ionising radiation; in comparison, the mean effective dose from a noncontrast CT brain scan is similar to that of a year's natural background radiation.

Perhaps the single most significant MRI sequence in the imaging of acute stroke is diffusion-weighted imaging (DWI). ${ }^{19} 20$ Fiebach et $a^{21}$ have previously demonstrated the improved sensitivity of DWI over CT. Barber et $a^{22}$ compared detection of early signs of cerebral ischaemia on CT and DWI in patients with acute disabling stroke within $6 \mathrm{~h}$ of symptom onset, using the ASPECT score. They concluded that CT and DWI were 'comparable for detecting and quantifying signs of cerebral ischaemia', although ASPECT scores were lower when assessed with DWI,
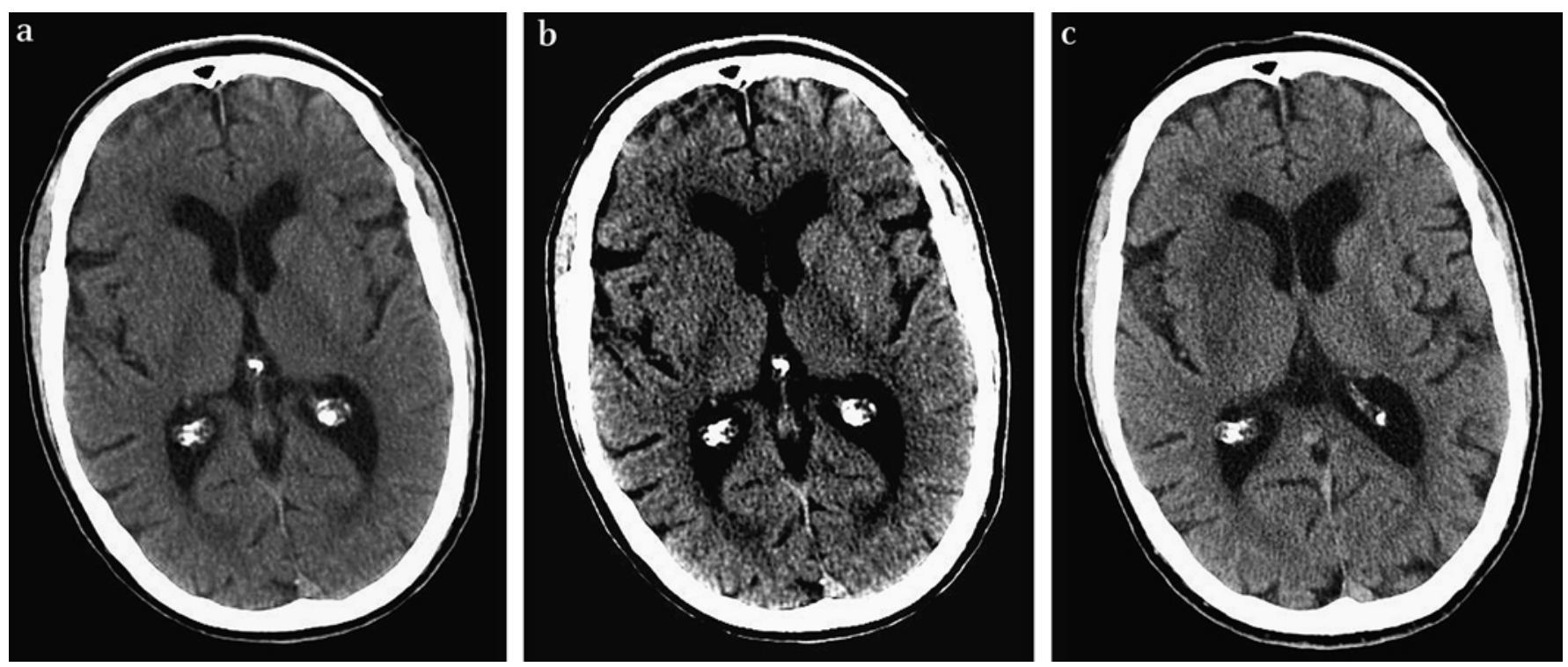

Figure 4 (A) Right basal ganglia infarct at $3 \mathrm{~h}$ using standard window width (W) 90 Houndsfield units (Hu) and centre length (L) $40 \mathrm{Hu}$. (B) Use of variable/narrow window levels accentuates the grey/white interface and makes the acute infarct more conspicuous, W 34 Hu, L 36 Hu. (C) Maturation of the right basal ganglia infarct at $18 \mathrm{~h}$. 
Figure 5 Hyperdensity within the lumen of the vessel-'hyperdense middle cerebral artery (MCA) sign'-is detected as $(A)$ linear hyperdensity within the proximal M1 segment of the MCA (black arrow) or (B) as a hyperdense 'dot' within the sylvian fissure (black arrow head).
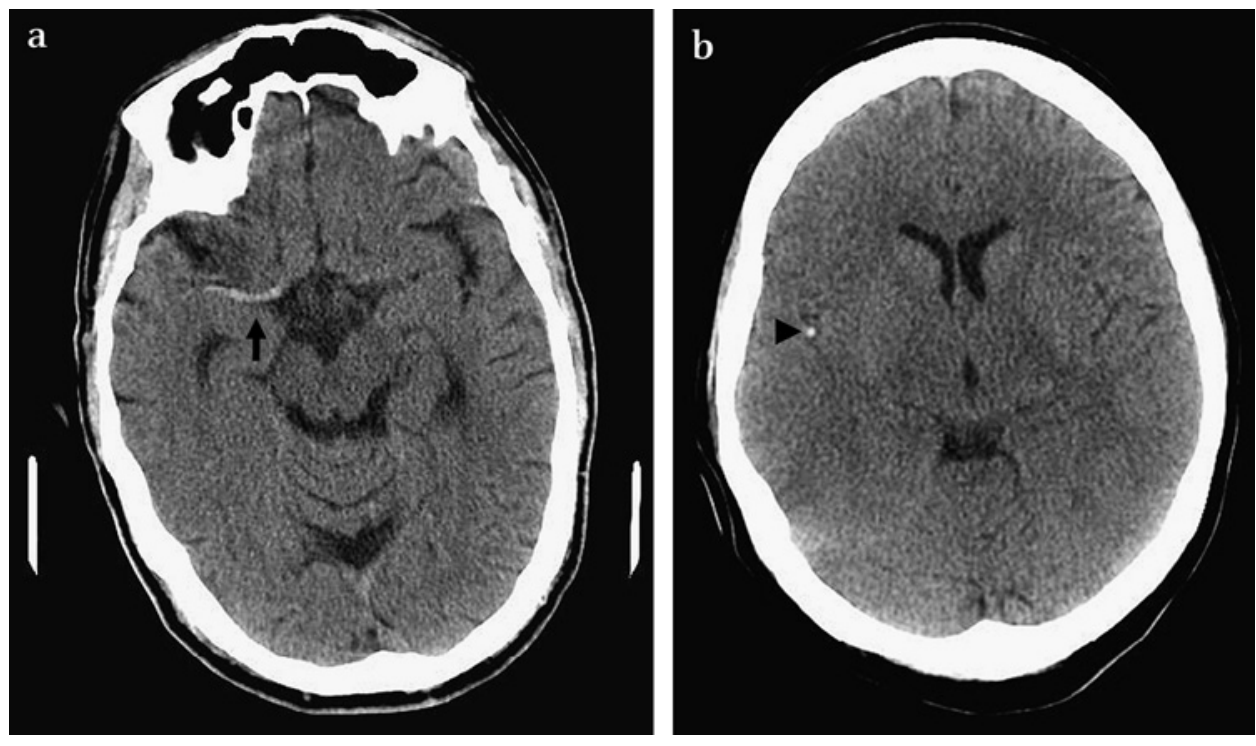

implying DWI to be more sensitive. In a prospective blinded study, Chalela et al $^{23}$ showed MR sensitivity of $83 \%$ vs $26 \%$ for CT in the diagnosis of any acute stroke; the MRI, however, encompassed both DWI and susceptibility-weighted images.

DWI uses the measurement of Brownian motion of molecules. As mentioned above, the cellular process during a cerebral infarct is rapid disruption of the sodium/potassium pump and influx of water molecules into the intracellular compartment-cytotoxic oedema. There is a resultant reduction in hydrogen ion diffusion ('restricted diffusion') and hence a low apparent diffusion coefficient (ADC), which manifests as a black area on the ADC map and as hyperintensity on DWI, (figure 7). ${ }^{24} 25$ Acquisition time for this sequence is under $1 \mathrm{~min}$ on most $1.5 \mathrm{~T} \mathrm{MR}$ systems, and detection of acute/hyperacute infarction is possible within minutes of the event. ${ }^{17}$ Diffusion restriction is not exclusive to acute cerebral infarcts; it can be seen in a variety of other conditions including cerebral abscess formation and encephalitis, highly cellular tumours such as lymphoma, haemorrhage, active demyelination (rarely) and following seizure activity. A review of clinical history and conformation (or lack of) to a vascular territory may aid differentiation.
Other MR sequences offer complementary information in the evaluation of patients with acute stroke. Multiplanar spinecho scans (T2-weighted and proton density imaging) identify the same early signs as those depicted on the non-contrast CT study but with increased sensitivity and specificity. ${ }^{26}$ Increased T2-weighted signal secondary to cytotoxic oedema results in loss of grey/white differentiation. Local sulcal effacement can be readily identified, and loss of normal arterial flow voids, depicting flow stasis distal to a thrombus, can be detected (figure 8). ${ }^{26}$ Many of these signs are not seen within the initial hours after infarction; as is the case on the noncontrast CT study, T2 hyperintensity may take several hours to develop.

Gradient-recalled echo imaging is highly susceptible to the paramagnetic effect of the blood product deoxyhaemoglobin, and hence this sequence is highly specific for identifying areas of microscopic and macroscopic haemorrhage (figure 9). ${ }^{27}$

Fluid-attenuated inversion recovery (FLAIR) MR, a heavily T2-weighted sequence, suppresses cerebral spinal fluid signal and shows other fluid with high conspicuity; hence intracranial haemorrhage is readily depicted. ${ }^{27} 28$ The development of signal
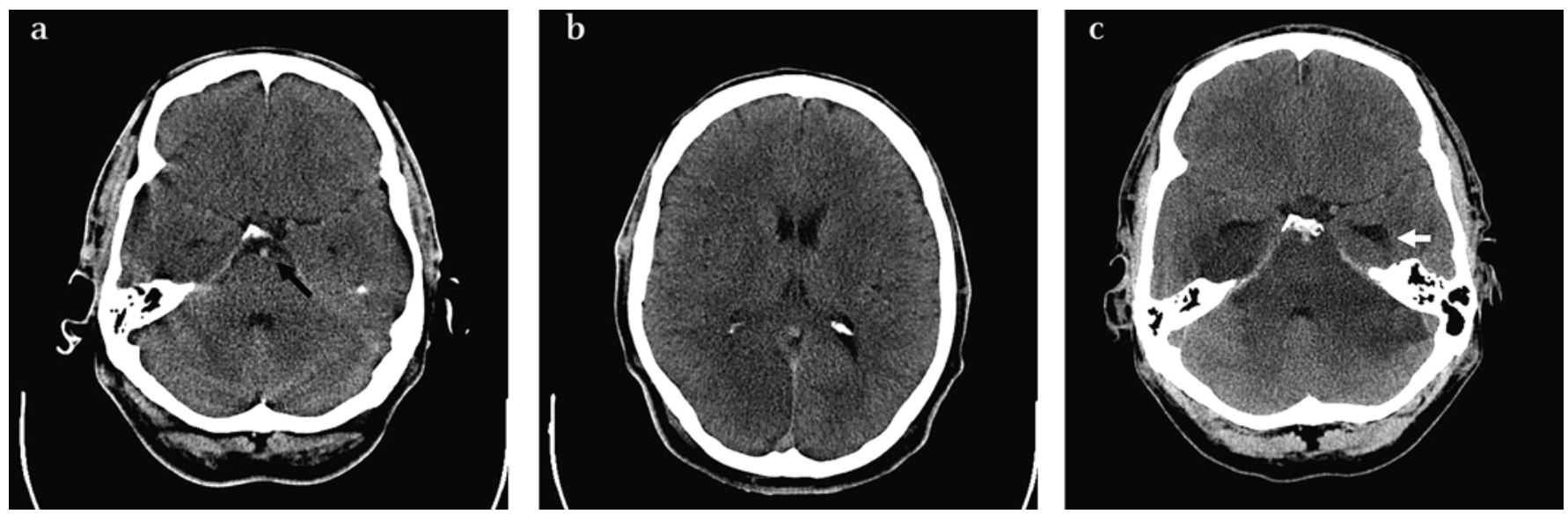

Figure 6 A patient with a history of anabolic steroid use and acute presentation with reduced level of consciousness. Presentation non-contrast CT (A) shows hyperdensity within the basilar artery consistent with thrombosis (black arrow); there is evidence of early right posterior cerebral artery territory infarction (B) with loss of grey/white differentiation. Follow-up imaging at $24 \mathrm{~h}$ shows extensive infarction within the brain stem extending into the middle cerebellar peduncles (C). The resultant white matter oedema results in partial fourth ventricular effacement and evolving hydrocephalus (note the temporal horn dilatation (white arrow)). 
Figure 7 Non-contrast CT (A) shows diffuse left hemispheric swelling and early subtle loss of grey/white differentiation within the middle cerebral artery (MCA) territory; note the hyperdense vessel in the left sylvian fissure (black arrow). Diffusionweighted imaging MR $(B)$ defines the acute MCA infarct with increased conspicuity, with hyperintensity throughout the left MCA territory consistent with diffusion restriction and a large acute infarct.
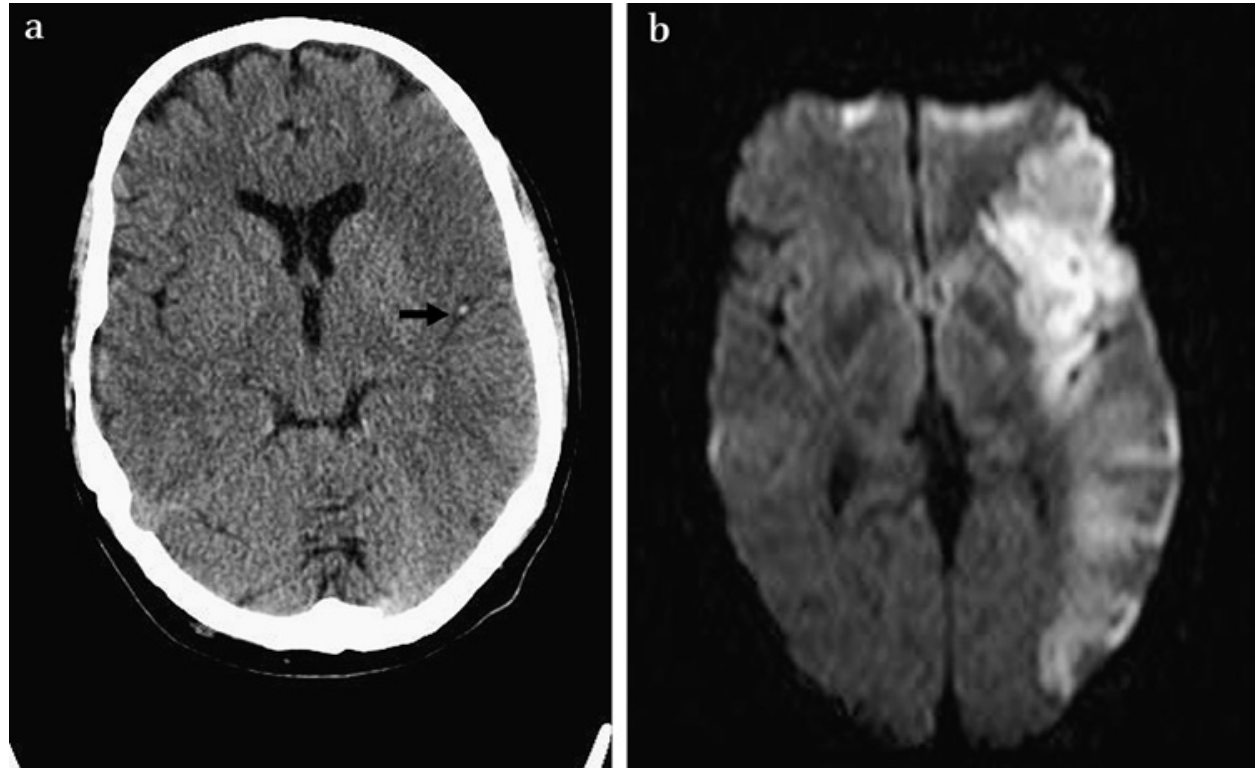

change within infarcted cortical or central grey matter is consistently seen earlier on FLAIR imaging (within $3 \mathrm{~h}$ ) than it is depicted with conventional spin-echo imaging. ${ }^{29} 30$

MRI does offer improved anatomical detail and increased sensitivity for acute infarct detection over CT. However, it is less readily available than CT and its usage in the acute stroke setting can be hampered by contraindications (such as cardiac prostheses) or confused patient status. In one study comparing CT and MRI in acute stroke, 45\% of 112 patients had 'contraindications' to MRI; reasons included medical instability, uncooperative patient or MR-specific exclusions. ${ }^{22}$

\section{Advanced CT and MRI techniques}

CT angiography (CTA) allows rapid non-invasive assessment of the intracranial and extracranial circulation at high resolution with multislice technology. By using a contrast bolus tracking

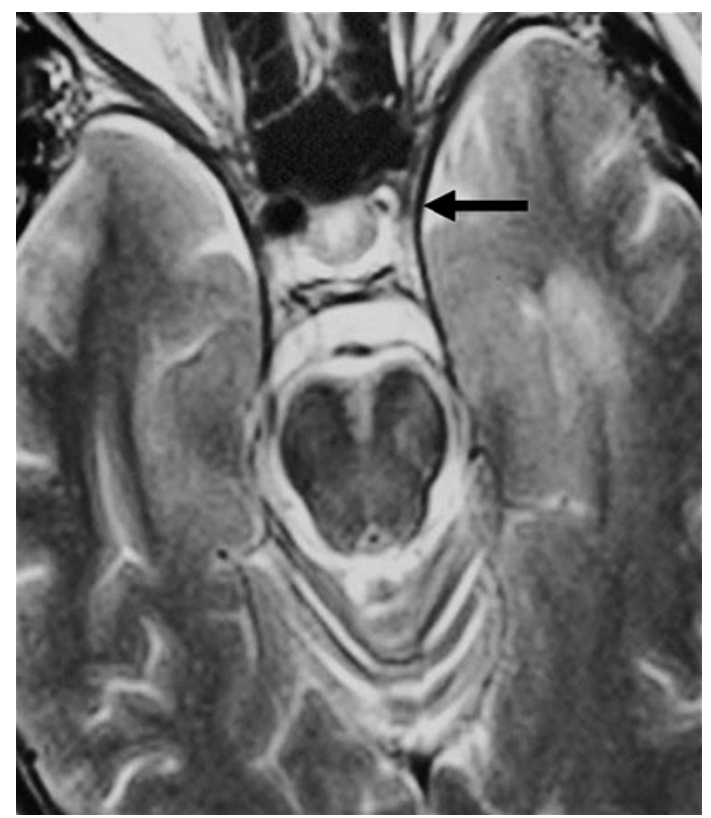

Figure 8 Axial T2-weighted MRI delineates loss of flow void within the left internal carotid artery (black arrow). Note the normal flow void within the right internal carotid artery. technique, a chosen volume (eg, circle of Willis or from the aortic arch to vertex) can be obtained within seconds. CTA permits assessment of arterial stenosis, occlusion (figure 10), craniocervical arterial dissection, and identification of stroke mimics such as arteriovenous malformations.

Magnetic resonance angiography (MRA) also allows noninvasive assessment of both the intracranial and extracranial vessels. Various techniques exist including both two-dimensional and three-dimensional time-of-flight (TOF), multiple overlapping thin slab acquisition (MOTSA) and contrastenhanced MRA. ${ }^{7}$ Assessment of the intracranial vasculature with TOF-MRA in the setting of acute stroke allows depiction of large-vessel occlusion. Guidance from the American Heart Association recommends that, in the acute setting, this should only be performed if it does not delay intravenous thrombolysis within $0-3 \mathrm{~h}$ of ictus or if intra-arterial thrombolysis or mechanical thrombectomy is being considered and this facility is available. $^{71}$ TOF-MRA is limited by its spatial resolution, and, as such, it's depiction of more distal vessel occlusion is inferior to conventional catheter digital subtraction angiography.

Where craniocervical arterial dissection is considered as the underlying aetiology, MRA assessment of the carotid and vertebral arteries is advised from the level of the aortic arch to the circle of Willis, and this can be easily added on to the initial brain MRI assessment. ${ }^{32}$ Spontaneous craniocervical arterial dissections account for only $2 \%$ of all ischaemic strokes, ${ }^{33}$ yet are responsible for up to $20 \%$ of such strokes in patients under 45 years of age. ${ }^{32}$ Routinely utilised sequences include axial T1 and T2 and MRA, either with or without contrast enhancement, and findings include detection of an intimal flap, loss of normal flow void, intraluminal high signal on T1 consistent with stasis, and classical tapering of the vessel lumen on MRA (figure 11). ${ }^{34}$ Some authors advocate use of axial T1 fat suppression to detect intraluminal haematoma; this sign, however, may not occur within the initial hours after ictus and may therefore be more relevant in the sub-acute setting. ${ }^{7}$ Conventional catheter digital subtraction angiography was previously the reference standard technique for evaluation of craniocervical dissection. However, the evolution of sensitive non-invasive techniques has superseded this technique in the routine setting; both MRA and CTA have been shown to be highly sensitive in the evaluation of this condition. ${ }^{33} 3536$ 
Figure 9 (A) Non-contrast CT performed $24 \mathrm{~h}$ after presentation with an acute left middle cerebral artery territory infarct shows low attenuation within the left head of the caudate nucleus and lentiform nucleus; high lentiform infarct was thought to represent probable micro-haemorrhage into the infarct. (B) A coronal gradientrecalled echo MRI study performed the same day clearly shows the presence of blood products at this site. attenuation within the centre of the left
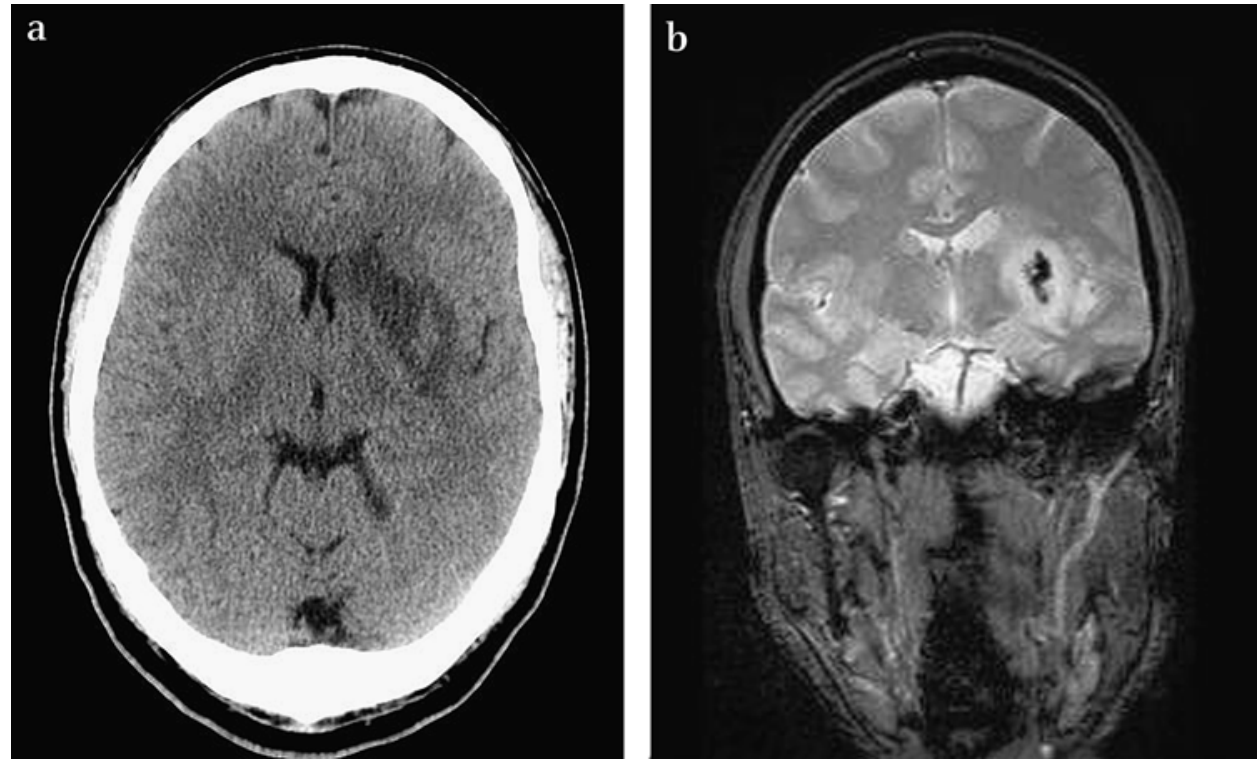

\section{DELINEATION OF THE ISCHAEMIC PENUMBRA}

Increasingly, multimodal imaging is being used to tailor optimal management strategies in the acute stroke setting. ${ }^{37-42}$ Advanced imaging allows assessment of the intracranial and extracranial vasculature and facilitates delineation of the status of cerebral perfusion, revealing the infarct core and the penumbra.

The ischaemic penumbra can be defined as 'ischemic tissue potentially destined for infarction but not yet irreversibly injured and the target of acute therapies'. ${ }^{43}$ The ischaemic
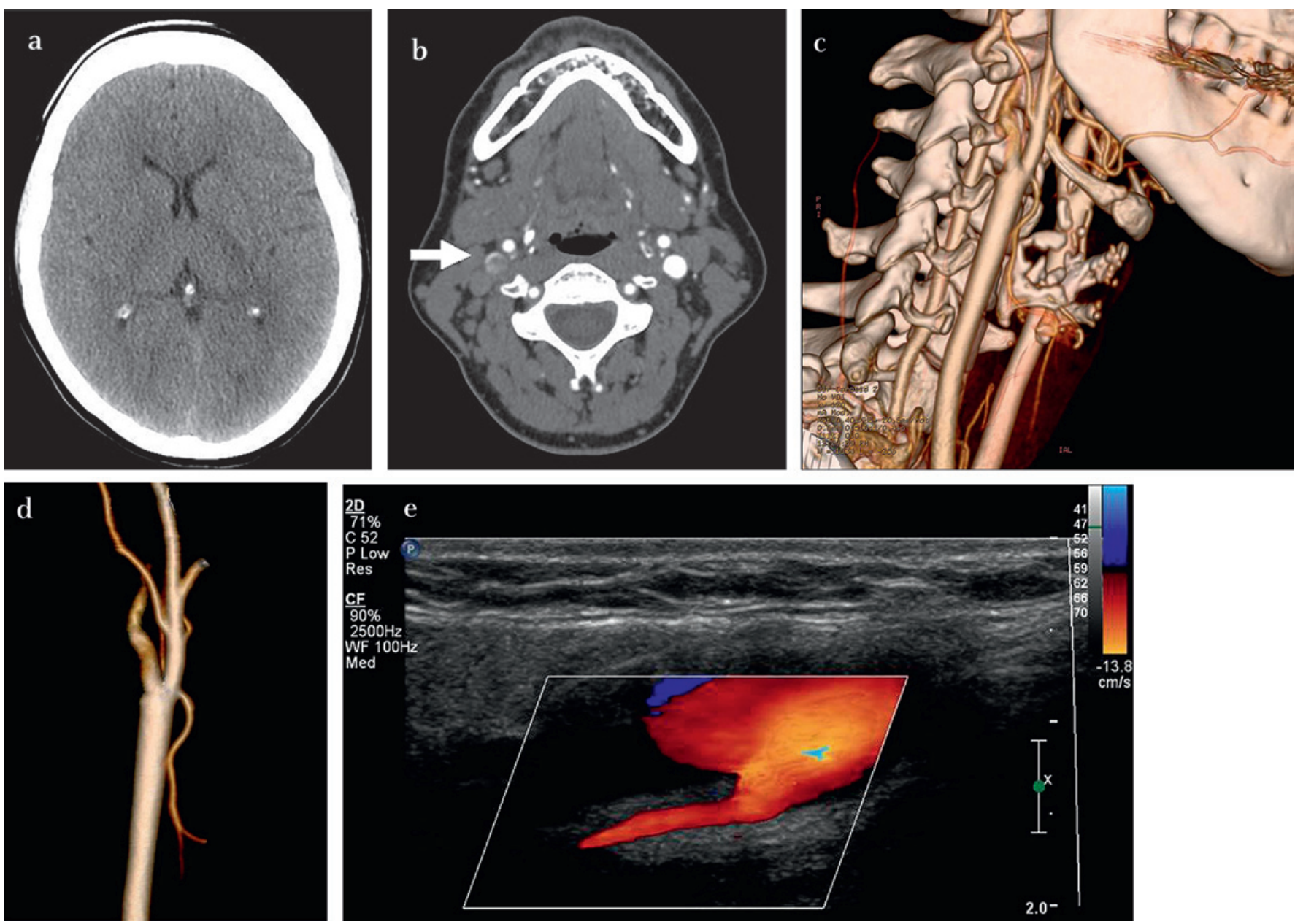

Figure 10 Patient with acute left arm weakness and neck pain. The presentation non-contrast CT (A) shows low attenuation within the right head of the caudate and lentiform nuclei. Axial CT angiography (CTA) source data (B) show the proximal right internal carotid artery occlusion (white arrow), and the dissection flap is confirmed on three-dimensional volume-rendered CTA imaging (C,D) and also on Doppler ultrasound (E). 

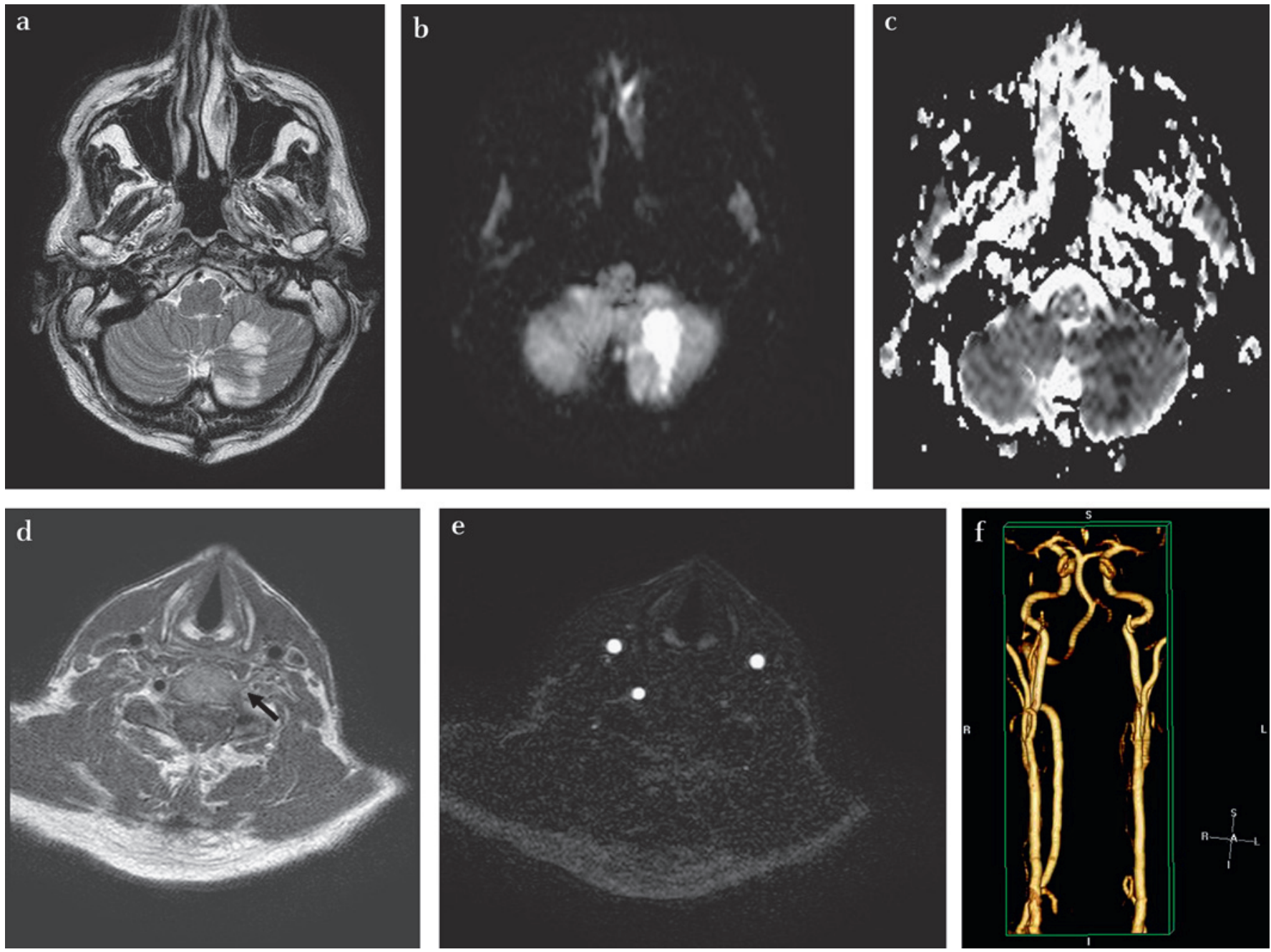

Figure 11 High-resolution T2 imaging of the posterior fossa (A) reveals an acute infarct within the left posterior inferior cerebellar artery territory in a young patient presenting with left-sided neck pain and unsteadiness of gait. Diffusion imaging shows increased diffusion (B) and low apparent diffusion coefficient $(C)$ consistent with diffusion restriction and acute infarction. Axial T1 imaging of the neck (D) confirms high T1 intraluminal signal within the left vertebral artery (black arrow), and (E) axial MR angiography (MRA) acquisition confirms this loss of left vertebral artery flow void. Maximum intensity projection reconstruction of three-dimensional time-of-flight MRA (F) clearly shows lack of flow in the left vertebral artery depicted as vessel absence, implying thrombus, although an underlying dissection cannot be excluded.

penumbra is therefore hypoperfused parenchyma in which neuronal electrical activity is disrupted but remains salvageable with restoration of blood flow; imaging evaluation of cerebral perfusion is hence critical in determination and calculation of the penumbra. The infarct core displays low cerebral blood flow (CBF), which is probably irreversible.

In July 2009, the Stroke Therapy Academic Industry Roundtable (STAIR) consortium recommended that 'future extended
Figure 12 Non-contrast CT (A) shows a large right middle cerebral artery infarct; the CT perfusion cerebral blood volume (B) highlights the extent of the infarct 'core'.
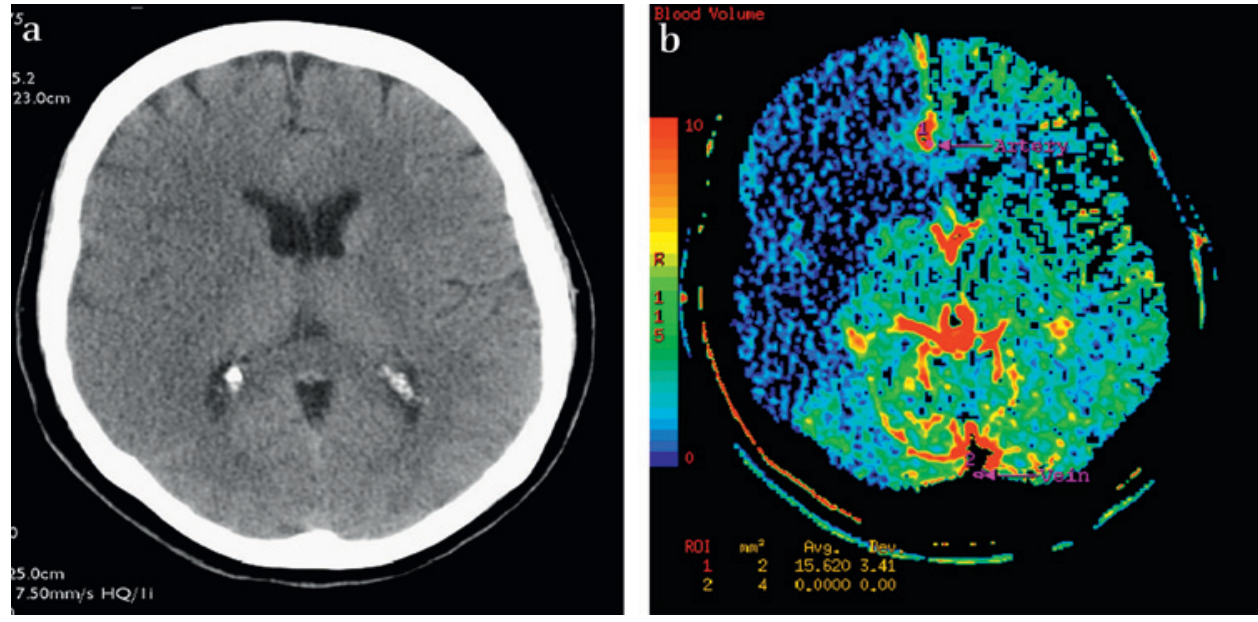

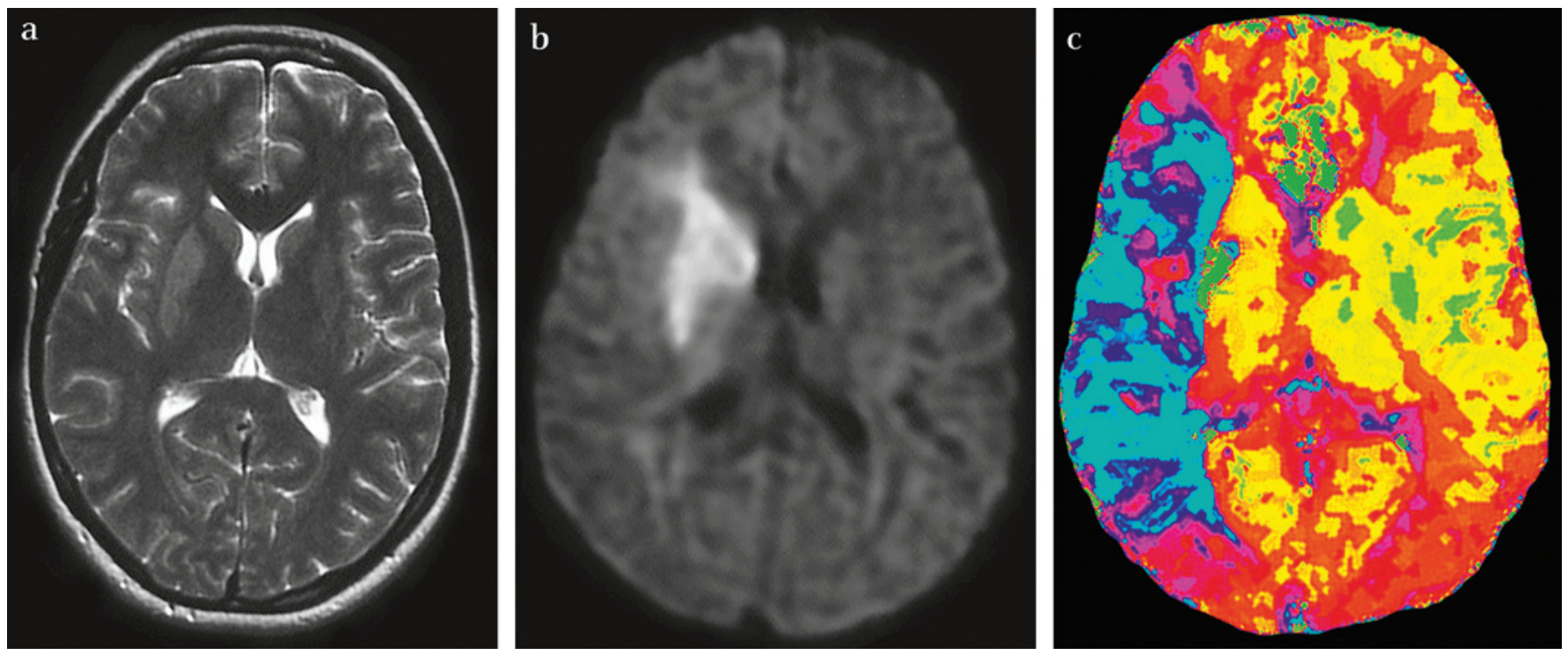

Figure $13 \mathrm{MRI}$ of an acute right middle cerebral artery (MCA) infarct at $4 \mathrm{~h}$. Axial T2-weighted image (A) shows subtle increased T2 signal within the right lentiform nucleus, diffusion-weighted imaging (B) shows the infarct core with improved conspicuity, while perfusion imaging (C) shows partial reperfusion of the infarct core but with profoundly reduced perfusion throughout the right MCA territory-the diffusion/perfusion mismatch representing potentially salvageable parenchyma.

time window trials initially should focus on selected patient groups most likely to respond to investigational therapies and that penumbral imaging is one tool that may identify such patients'. ${ }^{4}$ Several imaging techniques have been used to define the ischaemic penumbra including positron emission tomography, CTand MRI. Access and availability of positron emission tomography are limited, and hence most work with regard to mapping of the penumbra is focused on CT and MRI acquisition. ${ }^{43}$

CT perfusion (CTP) imaging uses a contrast bolus tracking technique (although this technique can also be performed with xenon inhalation). In contrast with $\mathrm{MR}$ perfusion, CTP offers both qualitative and quantitative measurements of $\mathrm{CBF}$, cerebral blood volume (CBV) and mean transit time (MTT). ${ }^{45}$ A series of images is acquired at a given level, usually a level that encompasses portions of the anterior, middle and posterior cerebral arteries-that is, at the level of the basal ganglia (figure 12). CBF/CBV mismatch with prolonged MTT is akin to the MR perfusion/ diffusion mismatch. When CTP is used, if the complete study includes a preliminary non-contrast CT brain scan, CTA (arch to vertex) and CTP, then the overall radiation dose is considerable. One recent study from Canada reviewed 95 case images on a 64slice CT scanner and showed a mean effective dose from baseline non-enhanced CT brain scan to be $2.7 \mathrm{mSv}$, while additional CTA and CTP took the mean effective dose to $13 \mathrm{mSv}$ - nearly five times the dose of the non-contrast study in isolation. ${ }^{46}$

$\mathrm{MR}$ perfusion imaging is most often performed using a bolus injection of gadolinium and $\mathrm{T}^{*}{ }^{*}$ susceptibility-weighted sequence. ${ }^{26} 47$ Images are acquired every $1-2 \mathrm{~s}$; as the contrast passes through the intracranial vasculature, there is a resultant signal drop-off, the degree of which is proportional to the CBF. This transient signal drop-off can be plotted on a time-intensity curve, from which various variables can be gleaned, including relative CBV (this is the area under the curve and would reduce in areas of hypoperfusion), relative CBF, MTT and time to peak. ${ }^{47} \mathrm{MR}$ perfusion is a qualitative study, and selected regions of interest are compared with the contralateral hemisphere. DWI depicts what is deemed to be the infarct core, and hence the 'diffusion/perfusion' mismatch potentially represents salvageable parenchyma (figure 13).

\section{NECK VESSEL ASSESSMENT}

Assessment of the extracranial vasculature is important when craniocervical dissection is considered as the underlying aetiology of stroke and also for assessment of vascular (predominantly carotid) stenosis. The NICE guidelines recommend that 'all people with suspected non-disabling stroke or transient ischaemic attack who, after specialist assessment, are considered as candidates for carotid endarterectomy should have carotid imaging within 1 week of onset of symptoms'. ${ }^{1}$ Doppler ultrasound is the most widely available, cheapest and minimally invasive test for this purpose, but CTA and MRA, as mentioned above, can also be used. In the acute stroke setting, the principal role of neck vessel imaging is to identify an underlying mechanism, such as arterial occlusion (secondary to either thrombus

\section{Key references}

- National collaborating centre for chronic conditions. Stroke: national clinical guidelines for diagnosis and the initial management of acute stroke and transient ischaemic attack (TIA). London: Royal College of Physicians, 2008.

- The National Institute of Neurological Disorders and Stroke rt-PA Stroke Study Group. Tissue plasminogen activator for acute ischaemic stroke. N Engl J Med 1995;333:1581-7.

- Hacke W, Kaste M, Bluhmki E, et al. Thrombolysis with alteplase 3 to 4.5 hours after acute ischemic stroke. $N$ Engl J Med 2008;359:1317-29.

- Barber PA, Demchuk AM, Zhang J, et al. Validity and reliability of a quantitative CT score in predicting outcome of hyper acute stroke before thrombolytic therapy. ASPECTS Study Group. Alberta Stroke Programme Early CT Score. Lancet 2000;355:1670-4.

- Saver JL, Albers GW, Dunn B, et al. Stroke Therapy Academic Industry Roundtable (STAIR) recommendations for extended window acute stroke therapy trials. Stroke 2009;40:2594-600. 


\section{Main messages}

- CT of the brain enables rapid assessment of patients with acute stroke in a safe environment and remains the modality of choice for initial assessment of acute stroke in most institutions.

- 'Time is brain'. Rapid clinical and imaging assessment of patients with acute stoke is crucial to optimising outcome.

- Physiological demonstration of an ischaemic penumbra may influence a decision for thrombolysis in patients presenting more than $3 \mathrm{~h}$ after symptom onset. Multimodal imaging and delineation of the penumbra (including CT or MR perfusion) is likely to guide future management strategies and will be pivotal in extension of potential therapeutic windows.

- MRI provides improved anatomical detail over CT and defines early ischaemic change with greater conspicuity. MRI is the preferred modality for assessment of posterior circulation infarcts and in the assessment of craniocervical arterial dissection.

- Assessment of the extracranial vasculature in patients with acute stroke may define the underlying stroke mechanism and can be performed non-invasively with Doppler ultrasound, CT angiography or MR angiography.

\section{Current research questions}

- What are the alternative imaging modalities for defining the ischaemic penumbra?

- How can identification of areas of programmed apoptosis be improved with current imaging modalities and is there a role for functional MRI?

- By what criteria and with which imaging modalities can the therapeutic window for thrombolysis be widened?

or dissection), or vessel stenosis, the depiction of which may influence therapy. ${ }^{7}$ Non-invasive imaging techniques for neck vessel assessment, including Doppler ultrasound, CTA and MRA, offer similar levels of accuracy, the choice of which is often therefore determined by local availability and preference.

\section{CONCLUSIONS}

Optimisation of acute stroke management pathways is ongoing. 'Time is brain', and hence early patient presentation and expeditious clinical and imaging assessment are paramount. Multimodal imaging techniques often offer complementary information, but, increasingly, protocols to extend the time to therapeutic intervention will be driven by the delineation of the ischaemic penumbra. Non-contrast CT as the initial imaging test remains the reference standard.

\section{MULTIPLE-CHOICE QUESTIONS (TRUE (T)/FALSE (F); ANSWERS AFTER THE REFERENCES)}

\section{Regarding diffusion imaging on MR:}

A. Diffusion restriction is specific to acute ischaemia

B. Diffusion restriction can be seen in cerebral abscess formation

C. Restricted diffusion does not occur within the first hour of acute infarction
D. An area of acute infarction is high signal on the ADC map

E. An area of acute infarction is low signal on diffusion

2. Regarding MRI in acute stroke:

A. Gradient-recalled echo MRI is insensitive to the detection of acute subarachnoid haemorrhage

B. Signal change is seen earlier on conventional dual echo imaging than FLAIR imaging

C. MRI is less sensitive in the detection of acute stroke than CT

D. DWI can be acquired in $<1$ min on most $1.5 \mathrm{~T}$ MR scanners

E. CT affords the same anatomical detail as MRI

\section{Spontaneous craniocervical arterial dissections:}

A. Account for only $2 \%$ of all ischaemic strokes

B. Are responsible for $40 \%$ of ischaemic strokes in patients under 45 years of age

C. Assessment with CTA is unreliable

D. Should not routinely be sought in young patients presenting with acute ischaemic stroke

E. Confirmation of flow void on axial MRI assessment is diagnostic

4. The Alberta Stroke Program Early CT (ASPECT) score:

A. Is less reliable than the one-third MCA rule at predicting MCA infarct extent and prognosis

B. Is a qualitative scoring system

C. An ASPECT score of $>7$ is associated with improved post thrombolysis outcome

D. Overall score is formulated by point summation depending on the number of areas displaying early ischaemic change

E. A normal CT brain scan with no evidence of ischaemic change would score zero points

\section{Regarding CT in the assessment of acute stroke:}

A. Multidetector technology permits image acquisition within minutes

B. Major current guidelines pertaining to thrombolysis accept non-contrast CT as sufficient basis for formulating decision to proceed to thrombolysis

C. The cumulative radiation dose of non-contrast CT, CTA and CT perfusion is negligible

D. Acute cerebral infarction results in vasogenic oedema

E. In the clinical setting, CT perfusion is most commonly performed with inhalation of xenon

Competing interests None.

Provenance and peer review Commissioned; externally peer reviewed.

\section{REFERENCES}

1. National Collaborating Centre for Chronic Conditions. Stroke: national clinical guidelines for diagnosis and the initial management of acute stroke and transient ischaemic attack (TIA). London: Royal College of Physicians, 2008.

2. Hatano S. Experience from a multicentre stroke register: a preliminary report. Bull World Health Organ 1976;54:541-53.

3. Adamson J, Beswick A, Ebrahim S. Is stroke the most common cause of disability? J Stroke Cerebrovasc Dis 2004;13:171-7.

4. The National Institute of Neurological Disorders and Stroke rt-PA Stroke Study Group. Tissue plasminogen activator for acute ischemic stroke. N Engl J Med 1995;333:1581-7.

5. Hacke W, Kaste M, Bluhmki E, et al. Thrombolysis with alteplase 3 to 4.5 hours after acute ischemic stroke. N Engl J Med 2008;359:1317-29.

6. Bluhmki E, Chamorro A, Dávalos A, et al. Stroke treatment with alteplase given $3 \cdot 0-4 \cdot 5 \mathrm{~h}$ after onset of acute ischaemic stroke (ECASS III): additional outcomes and subgroup analysis of a randomised controlled trial. Lancet Neurol 2009:8:1095-102.

7. Latchaw RE, Alberts MJ, Lev MH, et al. Recommendations for imaging of acute ischemic stroke: a scientific statement from the American Heart Association. 
American Heart Association Council on Cardiovascular Radiology and Intervention, Stroke Council, and the Interdisciplinary Council on Peripheral Vascular Disease. Stroke 2009; $\mathbf{4 0 : 3 6 4 6 - 7 8}$

8. Wardlaw JM, Mielke 0 . Early signs of brain infarction at CT: observer reliability and outcome after thrombolytic treatment-systematic review. Radiology

2005:235:444-53.

9. Tomura N, Uemura K, Inugami A, et al. Early CT finding in cerebral infarction: obscuration of the lentiform nucleus. Radiology 1988;168:463-7.

10. Truwit CL, Barkovich AJ, Gean-Marton A, et al. Loss of the insular ribbon: another early CT sign of acute middle cerebral artery infarction. Radiology 1990;176:801-6.

11. Lev MH, Farkas J, Gemmete JJ, et al. Acute stroke: improved nonenhanced CT detection-benefits of soft-copy interpretation by using variable window width and center level settings. Radiology 1999;213:150-5.

12. Hacke W, Kaste $\mathbf{M}$, Fieschi $\mathrm{C}$, et al. Intravenous thrombolysis with recombinant tissue plasminogen activator for acute hemispheric stroke: the European Cooperative Acute Stroke Study (ECASS). JAMA 1995;274:1017-25.

13. Barber PA, Demchuk AM, Zhang J, et al. Validity and reliability of a quantitative computed tomography score in predicting outcome of hyperacute stroke before thrombolytic therapy. ASPECTS Study Group. Alberta Stroke Programme Early CT Score. Lancet 2000:355:1670-4.

14. Pexman JH, Barber PA, Hill MD, et al. Use of the Alberta Stroke Program Early CT Score (ASPECTS) for assessing CT scans in patients with acute stroke. AJNR Am J Neuroradiol 2001:22:1534-42.

15. Hill MD, Rowley HA, Adler F, et al. PROACT-II InvestigatorsSelection of acute ischemic stroke patients for intra-arterial thrombolysis with pro-urokinase by using ASPECTS. Stroke 2003:34:1925-31.

16. Demchuk AM, Hill MD, Barber PA, et al. NINDS rtPA Stroke Study Group, NIH. Importance of early ischemic computed tomography changes using ASPECTS in NINDS rtPA Stroke Study. Stroke 2005;36:2110-15.

17. Schellinger PD, Fiebach JB, Hacke W. Imaging-based decision making in thrombolytic therapy for ischemic stroke: present status. Stroke 2003:34:575-83.

18. Barber PA, Demchuk AM, Hudon ME, et al. Hyperdense sylvian fissure MCA "dot" sign: a CT marker of acute ischemia. Stroke 2001;32:84-8.

19. Schellinger PD, Fiebach JB, Jansen 0, et al. Stroke magnetic resonance imaging within 6 hours after onset of hyperacute cerebral ischemia. Ann Neurol 2001;49:460-9.

20. Chung SP, Ha YR, Kim SW, et al. Diffusion-weighted MRI as a screening tool of stroke in the ED. Am J Emerg Med 2002;20:327-31.

21. Fiebach JB, Schellinger PD, Jansen 0 , et al. CT and diffusion-weighted MR imaging in randomized order: diffusion-weighted imaging results in higher accuracy and lower interrater variability in the diagnosis of hyperacute ischemic stroke. Stroke 2002:33:2206-10.

22. Barber PA, Hill MD, Eliasziw M, et al. Imaging of the brain in acute ischaemic stroke: comparison of computed tomography and magnetic resonance diffusionweighted imaging. J Neurol Neurosurg Psychiatry 2005;76:1528-33.

23. Chalela JA, Kidwell CS, Nentwich LM, et al. Magnetic resonance imaging and computed tomography in emergency assessment of patients with suspected acute stroke: a prospective comparison. Lancet 2007;369:293-8.

24. Minematsu K, Li L, Fisher M, et al. Diffusion-weighted magnetic resonance imaging: rapid and quantitative detection of focal brain ischemia. Neurologr 1992; 42:235-40.

25. Rowley HA, Grant PE, Roberts TP. Diffusion MR imaging. Theory and applications. Neuroimaging Clin N Am 1999:9:343-61.

26. Provenzale JM, Jahan R, Naidich TP, et al. Assessment of the patient with hyperacute stroke: imaging and therapy. Radiology 2003;229:347-59.

27. Kidwell CS, Chalela JA, Saver JL, et al. Comparison of MRI and CT for detection of acute intracerebral hemorrhage. JAMA 2004;292:1823-30.

28. Makkat S, Vandevenne JE, Verswijvel G, et al. Signs of acute stroke seen on fluidattenuated inversion recovery MR imaging. AJR Am J Roentgenol 2002;179:237-43
29. Noguchi $\mathbf{K}$, Ogawa $\mathrm{T}$, Inugami $\mathrm{A}$, et al. MRI of acute cerebral infarction: a comparison of FLAIR and T2-weighted fast spin-echo imaging. Neuroradiology 1997:39:406-10

30. Kamran S, Bates V, Bakshi R, et al. Significance of hyperintense vessels on FLAIR MRI in acute stroke. Neurology 2000;55:265-9.

31. Ohue S, Kohno K, Kusunoki K et al. Magnetic resonance angiography in patients with acute stroke treated by local thrombolysis. Neuroradiology 1998:40:536-40.

32. Thanvi B, Munshi SK, Dawson SL, et al. Carotid and vertebral artery dissection syndromes. Postgrad Med J 2005;81:383-8.

33. Schievink WI. Spontaneous dissection of the carotid and vertebral arteries. N Engl J Med 2001;344:898-906.

34. Provenzale JM. MRI and MRA for evaluation of dissection of craniocerebral arteries: lessons from the medical literature. Emerg Radiol 2009;16:185-93.

35. Elijovich L, Kazmi K, Gauvrit JY, et al. The emerging role of multidetector row CT angiography in the diagnosis of cervical arterial dissection: preliminary study. Neuroradiology 2006;48:606-12

36. Vertinsky AT, Schwartz NE, Fischbein NJ, et al. Comparison of multidetector CT angiography and MR imaging of cervical artery dissection. AJNR Am J Neuroradiol 2008;29:1753-60.

37. Thomalla G, Schwark C, Sobesky J, et al. Outcome and symptomatic bleeding complications of intravenous thrombolysis within 6 hours in MRI-selected stroke patients: comparison of a German multicenter study with the pooled data of ATLANTIS, ECASS, and NINDS tPA trials. Stroke 2006:37:852-8.

38. Wintermark M, Meuli R, Browaeys P, et al. Comparison of CT perfusion and angiography and MRI in selecting stroke patients for acute treatment. Neurology 2007:68:694-7.

39. Gasparotti R, Grassi M, Mardighian D, et al. Perfusion CT in patients with acute ischemic stroke treated with intra-arterial thrombolysis: predictive value of infarct core size on clinical outcome. AJNR Am J Neuroradiol 2009;30:722-7.

40. Schellinger PD, Thomalla G, Fiehler J, et al. MRI-based and CT-based thrombolytic therapy in acute stroke within and beyond established time windows: an analysis of 1210 patients. Stroke 2007:38:2640-5.

41. Yoo AJ, Verduzco LA, Schaefer PW, et al. MRI-based selection for intra-arterial stroke therapy: value of pretreatment diffusion-weighted imaging lesion volume in selecting patients with acute stroke who will benefit from early recanalization. Stroke 2009;40:2046-54.

42. Ogawa A, Mori E, Minematsu K, et al. Randomized trial of intraarterial infusion of urokinase within 6 hours of middle cerebral artery stroke: the middle cerebral artery embolism local fibrinolytic intervention trial (MELT) Japan. Stroke 2007;38:2633-9.

43. Fisher M, Ginsberg M. Current concepts of the ischemic penumbra. Stroke 2004:35:2657-8

44. Saver JL, Albers GW, Dunn B, et al. STAIR VI Consortium. Stroke Therapy Academic Industry Roundtable (STAIR) recommendations for extended window acute stroke therapy trials. Stroke 2009;40:2594-600.

45. Cianfoni A, Colosimo C, Basile M, et al. Brain perfusion CT: principles, technique and clinical applications. Radiol Med 2007:112:1225-43.

46. Mnyusiwalla A, Aviv RI, Symons SP. Radiation dose from multidetector row CT imaging for acute stroke. Neuroradiology 2009;51:635-40.

47. Jäger HR. Diagnosis of stroke with advanced CT and MR imaging. Br Med Bull 2000:56:318-33.

\section{ANSWERS}

1. (A) $\mathrm{F}$; (B) $\mathrm{T}$; (C) $\mathrm{F}$; (D) $\mathrm{F}$; (E) $\mathrm{F}$

2. (A) $\mathrm{F} ;(\mathrm{B}) \mathrm{F} ;$ (C) $\mathrm{F}$; (D) $\mathrm{T}$; (E) $\mathrm{F}$

3. (A) $\mathrm{T}$; (B) $\mathrm{F}$; (C) $\mathrm{F}$; (D) $\mathrm{F}$; (E) $\mathrm{F}$

4. (A) $F$; (B) F; (C) $T$; (D) F; (E) $F$

5. (A) $\mathrm{F}$; (B) $\mathrm{T}$; (C) $\mathrm{F}$; (D) $\mathrm{F}$; (E) $\mathrm{F}$ 